\title{
FRAGMENTER LE CORPS, FRAGMENTER LES DROITS
}

Assumpta Sabuco i Cantó, traduit de l'espagnol par Salvador Juan

\author{
L'Harmattan | «L'Homme et la société »
}

2017/1 n²03-204 | pages 113 à 138

ISSN 0018-4306

ISBN 9782343129921

Article disponible en ligne à l'adresse :

https://www.cairn.info/revue-l-homme-et-la-societe-2017-1-page-113.htm

\section{Pour citer cet article :}

Assumpta Sabuco i Cantó, "Fragmenter le corps, fragmenter les droits », L'Homme et la société 2017/1 ( $\left.\mathrm{n}^{\circ} 203-204\right), \mathrm{p} .113-138$.

DOI 10.3917/lhs.203.0113

Distribution électronique Cairn.info pour L'Harmattan.

(c) L'Harmattan. Tous droits réservés pour tous pays.

La reproduction ou représentation de cet article, notamment par photocopie, n'est autorisée que dans les limites des conditions générales d'utilisation du site ou, le cas échéant, des conditions générales de la licence souscrite par votre établissement. Toute autre reproduction ou représentation, en tout ou partie, sous quelque forme et de quelque manière que ce soit, est interdite sauf accord préalable et écrit de l'éditeur, en dehors des cas prévus par la législation en vigueur en France. Il est précisé que son stockage dans une base de données est également interdit. 


\title{
Fragmenter le corps, fragmenter les droits*
}

\author{
Assumpta SABUCO I CANTÓ \\ Universidad de Sevilla
}

En avril 2017, Anthropology Today consacrait un numéro spécial au capitalisme et à la magie. Dans un éditorial suggestif, Timothy de Waal Malefyt décrivait l'intérêt d'associer ces termes puisque the articles in this issue argue for treating magic not as an object of study but as a subject of cultural forms, processes and practices, worthy of "magical" investigation in their own right ${ }^{1}$. La technologie intelligente pourrait remplir, dans cette relation, une triple fonction. Tout d'abord, générer des significations dans les objets qui lui confèrent charme et vérité : le réseau d'affects que nous éprouvons pour les belles choses nous entourant (TV, tablettes, mobiles, annonces, etc.) et qui sont insérés dans ce que Featherstone appelle The estheticization of everyday life $e^{2}$. Deuxièmement, fonctionner magiquement puisque nous ne connaissons pas les processus qui rendent opérationnelle la technologie intelligente, mais que nous croyons fidèlement en elle. Troisièmement, jouer un rôle important qui affecte non seulement le marché financier, mais encore la plupart des espaces sociaux en produisant des idéaux sociaux avec lesquels nous comparons la réalité.

On retrouve ce lien entre capitalisme et magie dans les études de nombreux auteurs qui ont analysé le pouvoir de la beauté dans la mondialisation,

1 Timothy de WaAl Malefyt, « Enchanting Technology », Anthropology Today, 33, 2 (2017), p. 2.

2 Mike Featherstone, Consumer culture and postmodernism, Los Ángeles, Sage, 2007 [2 éd. ; $1^{\text {re }}$ éd. : 1991]. 
l'émergence d'industries du cosmétique, la prolifération des cliniques de chirurgie esthétique, la floraison des publications spécialisées dans la mode, l'abondance des images changeantes qui sont rapidement intériorisées comme « besoins » pour l'épanouissement personnel.

Dans un premier temps, Alfred Gell ${ }^{3}$, et plus récemment Brian Moeran ${ }^{4}$, ont nommé ces nouvelles productions culturelles «technologies de l'enchantement ». Les études sur les valeurs, les mythes et les croyances de ces formes de la production corporelle avec des spécialistes, avec des rituels et des images spécifiques, occultent cependant la grande fragmentation qui transforme le corps des femmes en marchandise. Une décomposition de l'intégrité corporelle avait déjà été notée par Le Breton dans l'ordre mécaniciste et l'ordre anatomique qui font prévaloir les parties du corps sur le tout. De son point de vue :

Écarté abstraitement de l'homme à la façon d'un objet, vidé de son caractère symbolique, le corps l'est aussi de sa dimension axiologique. Il est aussi dépouillé de son halo imaginaire ${ }^{5}$.

Le propos de cet article est d'analyser les représentations, les pratiques et les idéaux, se développant de nos jours, sur les corps des femmes pour réfléchir sur l'une de ses caractéristiques les plus importantes: la fragmentation. Notre position articule deux traditions : d'une part, le regard sur la dislocation de ces identités magiques construites à travers l'esthétique et le culte du corps ; d'autre part, l'introduction des technologies imaginaires dans les luttes sur les droits du corps dans une perspective féministe. Les « vies qui méritent d'être vécues » ou «les vies dignes d'être pleurées » ${ }^{6}$

3 Alfred Gell, Art and Agency: An Anthropological Theory, Oxford-New York, Oxford University Press - Clarendon Press, 1998.

Alfred GELL, "The Enchantment of Technology and the Technology of Enchantment ", in Jeremy Coote \& Anthony Shelton (eds), Anthropology, Art and Aesthetics, Oxford, Clarendon Press, 1992, p. 40-66.

4 Brian Moeran, Magic of Fashion: Ritual Commodity, Glamour, Walnut Creek, Left Coast Press (Anthropology \& Business 3), 2015.

5 David Le Breton, Anthropologie du corps et modernité, Paris, PUF (Sociologie d'aujourd'hui), 1990, p. 230-231.

6 Judith ButLer, Marcos de guerra. Las vidas lloradas, trad. B. Moreno Carrillo, Barcelona, Paidos, 2010 [texte original : Frames of War. When Is Life Grievable?, 2009]. 
transforment l'esthétique et la maternité en cadres symboliques efficaces sur lesquels s'appuyer pour revendiquer des droits pour les uns et les autres.

\section{La magie de la fragmentation des corps}

La beauté est l'élément le plus important par lequel nous jugeons notre corps et le corps des autres. "Être belle » est une obligation que nous assumons avec satisfaction pour les promesses implicitement liées aux codes esthétiques dominants. Nous croyons que l'apparence de notre corps nous assurera de bonnes rencontres sexuelles, un meilleur travail, une vie de famille heureuse. Nous nous efforçons de ne pas prendre de poids, de changer notre garde-robe à chaque saison, de nous appliquer des crèmes et des substances, ce qui nous prend beaucoup de temps et d'argent.

Comme l'a noté Naomi Wolf dans les années 1990, le mythe de la beauté a remplacé la mystique féminine des années 1940 et 1950 lorsque l'injonction à être une bonne épouse et une bonne mère opprimait les femmes. Si donc les objets de la maison - et surtout ceux d'ordre électrodomestique - présentaient une image enviable de ce dont une femme avait besoin pour être heureuse - en plus de la donnée de base que constitue un mari -, aujourd'hui, c'est notre propre corps qu'il nous faut façonner pour nous sentir nous-mêmes, dans la concurrence sexuelle et pour réussir professionnellement. Notre corps a cessé d'être un élément de plaisir et de connaissance pour devenir un matériau de transformation duquel dépendent de multiples aspects sociaux : le couple, la vie professionnelle, le prestige.

Au cours du $\mathrm{XX}^{\mathrm{e}}$ siècle, les revues de mode, la publicité et le cinéma convertirent le "glamour » et l'élégance en idéaux esthétiques. Les aspirations à l'ascension sociale restèrent liées à l'image des femmes appartenant aux classes sociales aisées. Vêtements et soins externes étaient des garanties de succès pour de nombreuses femmes à travers le mariage. Il ne s'agissait plus d'offrir un esprit de sacrifice en obtenant une taille de guêpe, mais de maintenir un corps sportif et sain.

Dans nos sociétés, la bonne apparence est une «obligation » pour qui prétend obtenir un bon emploi ou le garder, faire un bon mariage ou atteindre une estime de soi davantage fondée sur un combat contre le temps, contre notre corporéité, que sur les « soins personnels ». En tant que stratégie de 
contrôle face à l'incertitude croissante qu'a provoquée la crise économique, les «soins » deviennent, de notre point de vue, un élément clef permettant une amélioration globale depuis des parties spécifiques du corps : les lèvres, les cils, les seins, la taille... L'exaltation de la santé assimilée à l'aspect physique devient un indicateur social qui touche et condamne les femmes. La prolifération des produits proposés pour certaines parties du corps, pour certains âges et pour les femmes avec des caractéristiques spécifiques est une tendance marchande très lucrative. Le nombre croissant d'opérations de chirurgie esthétique et d'offres de voyages de bien-être et de transformation physique, créant un nouveau tourisme de la santé, sont un signe très clair du degré d'intériorisation par les femmes de ces représentations et de combien elles les convertissent en pratiques médicales, auparavant occasionnelles ou liées à certaines classes sociales, en routines plus ou moins prégnantes mais qui affectent toutes les femmes également.

De la même manière, sont de plus en plus nombreux les préjugés idéologiques qui se développent autour d'une maternité morcelée. D’une part, l'utérus, le fœtus, les ovules ou même le lait maternel sont plus valorisés que l'expérience singulière de chaque femme. D’autre part, être mère tend à devenir une expérience à vivre depuis la « naturalité » de notre corps dans une confrontation claire avec la médicalisation croissante de la naissance, mais où les chances de grossesse s'étendent temporellement grâce à la technologie. Pour modifier ou implanter un embryon dans un utérus, on a dépassé les contraintes de l'âge et il y existerait un horizon illimité pour devenir mère.

Dans cette paradoxale tension entre la technicisation de la maternité et l'exaltation de cette dernière comme désir, la plus grande dichotomie réalisée dans le corps de la femme enceinte a été liée au fort controversé projet de loi présenté le 23 novembre 2013 par le ministre Gallardón, projet prétendant garantir la protection des droits des «plus faibles : ceux qui sont conçus et non encore nés ». Cela entraînait un retour à la pénalisation par l'abrogation du droit des femmes à avorter - sauf en cas de risque sérieux pour la vie de la mère ou si la grossesse résultait d'un viol. L'argumentaire accusait la loi de 2010, adoptée par le $\mathrm{PSOE}^{7}$ et toujours en vigueur, d’opérer un déséquilibre

7 Parti socialiste ouvrier espagnol (NdT). 
entre les droits de la mère et ceux dont serait titulaire «l'enfant à naître », en privant ce dernier de protection dans les 14 premières semaines de la grossesse. Par l'effet de la magie, le corps de la femme et le corps du non-né devenaient des entités juridiques distinctes, séparées, voire aux intérêts contradictoires, dont l'existence ne devait rien à une quelconque intervention masculine. Cela montre comment la segmentation dans la construction sociale du corps masculin n'use pas de la même dichotomie.

Les hommes se sont intégrés en tant qu'objets dans les industries de la consommation cosmétiques avec des différences qui doivent être signalées. La corrélation entre les attributs de la masculinité et les produits cosmétiques par leur taille, leur couleur et leur forme souligne l'efficacité et la fonctionnalité des crèmes. Si on les compare en termes de rentabilité, ces crèmes requièrent moins de temps que celles destinées aux femmes. De ces produits, c'est l'idée d'entretien du corps et non de soin qui prime, et ce par le biais de l'idée de rapidité et de vigueur. Il s'agit d'une valeur supérieure qui se reflète également dans les banques dites du sperme qui, par une intéressante inversion symbolique, conservent pourtant également des ovules.

Loin d'être tous comparables, les fragments corporels qu'utilise le marché accentuent l'inégalité structurelle qui légitime la domination des hommes face aux femmes, la meilleure signification et la plus grande pertinence de certains produits sur les autres. Le débat sur la maternité de substitution en Espagne met en évidence les doutes sur une pratique de plus en plus courante dans notre pays, qui a été a été "publicisée » par les médias dans leur quête du spectaculaire. Un exemple du pouvoir de séduction de cette conquête corporelle est le cas du chanteur Miguel Bosé annonçant en 2011 avoir deux enfants auxquels se sont ajoutés deux autres en seulement sept mois. Son "Papatour " a duré deux ans et demi et a suscité une large adhésion du public. Bien que le chanteur ait revendiqué son désir de paternité dans un magazine d'homosexuels, il n'a jamais voulu entrer dans les débats juridiques au sujet de son droit à louer des «mères porteuses » parce qu'il avait des enfants comme n'importe quel citoyen. 


\section{Des corps réels aux corps imaginés}

La substitution progressive des corps réels par les corps imaginés devient évidente si l'on considère l'importance des mannequins qui occupent une place centrale dans le monde de la vente de vêtements. Si, dans le passé, le prestige a été associé au fait de pouvoir se faire faire des costumes sur mesure, aujourd'hui c'est l'ajustement au poids et les marques qui engendrent la distinction. La disproportion entre les référents masculins et féminins dans les vitrines reflète la pression supérieure sur le genre féminin en vue d'atteindre certains canons pourtant éloignés de la réalité corporelle de nombreuses femmes.

L'extériorité de notre corps atteint des niveaux semblables à ceux d'un objet quelconque sur le marché ; il s'agit de se "sentir bien » et de montrer une chair lisse, nécessitant de plus en plus de soins : depuis l'aspect de la peau, les yeux, les cheveux et dans l'ensemble des parties les plus visibles du corps, jusqu'aux plus invisibles comme la transpiration ou la texture de l'épiderme. L'industrie renforce l'utilisation massive de prolongements pour les cheveux, les lentilles de couleur, les collants anticellulite, les soutiensgorge affermissant les seins de manière plus efficace que les "trucs » traditionnels - les anciennes augmentations de papiers ou de coton-, les crèmes d'allongement et d'épaississement des cils, du maquillage ou du rouge à lèvres indestructibles, des produits aux potentialités magiques pour éliminer les impuretés ou les défauts en quelques minutes.

Au développement de l'industrie des cosmétiques se sont ajoutées des possibilités de transformation plus permanente dépendent de la chirurgie et des interventions médicales. Les entreprises spécialisées dans l'esthétique y apportent une large part des bénéfices dérivés de cette nouvelle technologie du soi qui augmente les chances de transformation de manière inquiétante. Les publicités pour la transformation du corps reposent sur la segmentation du corps, en particulier du corps féminin. La distance entre les corps imaginés et les corps réels s'accentue à l'avantage des premiers.

Les changements que permet la technologie servent également à rendre les individus responsables de leur propre apparence en consolidant certains canons esthétiques et des coutumes qui bénéficient à certains secteurs économiques et politiques. Il n'est pas étonnant que les États présentent, au 
moins au niveau formel, comme l'une de leurs priorités le soin de la santé de leurs citoyens. Ne pas fumer, manger sainement, dormir suffisamment, deviennent des principes implicites, qui acquièrent de plus en plus d'évidence normative. En déléguant la responsabilité aux individus - comme le montrent les slogans publicitaires - se renforce la croyance, la conviction, de ce que nous devons faire de notre propre corps. Dans ce combat pour l'attention au corps, les opérations - du nez, des pommettes, des fesses - deviennent un processus sans limite d'âge et sans danger apparent, malgré les exigences croissantes face aux erreurs ou aux problèmes liés à ces pratiques.

Il est intéressant de noter que la langue elle-même fait partie du nouveau cadre idéologique de la beauté. "Nous nous faisons » une réduction du ventre ou «nous nous retirons » les varices ou les poches sous les yeux. Les chances d'acquérir un corps parfait sont sexuées puisque ce sont surtout les femmes qui s'offrent de tels services.

\section{La fragmentation esthétique comme mécanisme d'oppression}

L'idéal d'un corps parfait agit sur l'imaginaire collectif comme un désir réalisable, ce qui explique les énormes dépenses personnelles, les sacrifices et les regards subjectifs envers soi-même qui renforcent ce qui a été appelé « culte du corps ». Le revers de cette construction dominante du corps, par laquelle être un yogourt et « avoir un corps Danone » sont étroitement liés, sont les conséquences inégales que le pouvoir de la beauté génère chez les femmes et les hommes. L'anorexie est son expression la plus dramatique et celle qui a suscité le plus grand nombre d'études féministes des années 1970. Le livre de Susie Orbach, Fat is a Feminist Issue ${ }^{8}$, illustre les nouvelles références et les problèmes associés au mythe de la beauté durant la seconde moitié du $\mathrm{XX}^{\mathrm{e}}$ siècle, ainsi que la consommation croissante de la première décennie du XXI ${ }^{\mathrm{e}}$ siècle.

L'obéissance de plus en plus grande des hommes aux diktats de la mode correspond à l'expansion du marché des industries de l'embellissement. Les nouvelles classifications, comme "métrosexuel», et les problèmes de

8 Susie Orbach, Fat is a Feminist Issue, Middlesex, Paddington Press, 1978. 
« vigorexie » ${ }^{9}$ renferment une double signification : d'une part, l'affirmation de la masculinité et, d'autre part, la transformation de celle-ci, avec une perspective positive à l'égard des formes et des usages liés à la féminité, tels que la promotion de l'épilation chez les hommes.

Dans cette modification des modèles de genre, un facteur décisif a été de faire de la beauté un signe de santé. Cela a permis d'augmenter le nombre de produits disponibles sur le marché pour contrecarrer les imperfections, éliminer les défauts, régénérer ou modifier tout ce que nous voulons sur notre corps. Plutôt que d'accepter nos caractéristiques corporelles et physiques, il s'agit de lutter pour atteindre un idéal social, d'avoir foi en une stratégie magique par laquelle le contrôle sur le corps assure la réalisation des fins socialement valorisées.

Dans cette "guerre » constante contre notre corps, la fragmentation permet de livrer des batailles partielles avec l'aide des produits que nous fournissent les puissantes industries cosmétiques et pharmaceutiques, des gymnases, des magasins de vêtements et des nouvelles chaînes pour les soins du corps. Des éléments naturels tels que le soleil, le vent, le froid ou la chaleur sont devenus des ennemis à combattre car ils perturbent notre équilibre. L'utilisation quotidienne de notre corps doit se préserver de l'usure par l'emploi de produits appropriés. D'où l'attention accrue à la santé de chacun des éléments qui nous composent : les mains, les yeux, les hanches, et même les pieds, que beaucoup de prothèses et d'offres spécialisées peuvent suppléer. On continue à faire grand cas d'éléments esthétiques qui limitent le mouvement des femmes, tels les talons aiguilles, mais dans n'importe quel magasin tous types d'aide pour en réduire les effets nocifs sont disponibles : semelles en silicone, adhésifs antidérapants, etc.

Les recettes magiques d'amincissement et les gymnases, les salles de spa contre le stress sont quelques-uns des compléments nécessaires dans ce processus d'entretien du corps ${ }^{10}$. Ce type d'attentions, ainsi que ses éléments

9 Mario JoRDI, «El músculo en el centro: masculinidades en los gimnasios contemporáneos », in J.M.V. Valcuende del Río, M.J.M. Macarro \& D. Alarcón Rubio (dir.), Estudios sobre diversidad sexual en Iberoamérica, actas de I Congreso internacional (Sevilla, junio 2012), Sevilla, Aconcagua Libros, 2013, p. 321-334.

10 Lourdes MÉNDEZ PÉREZ, Recetarios mágico-científicos al servicio de la estética de la delgadez: cuerpos de mujeres, cuerpos de hombres, [Santiago de Compostela], Museo do Pobo Galego, 1995. 
d'accompagnement se sont étendus avec la consécutive intériorisation de leur caractère vital, en cela que nous sommes responsables de notre négligence. Il s'agit de renforcer la veille et les efforts sur l'individuel, d'exercer un suivi continu de soi-même. Assumer et gérer le corps devient particulièrement préoccupant dans les étapes où l'âge, la grossesse ou les changements métaboliques imposent des transformations qu'il faut limiter pour se réadapter aux impératifs esthétiques. Dans ces technologies de genre $^{11}$ se renforce le refus des modifications corporelles par une illusion de jeunesse permanente.

La prolifération des éléments à capacité régénératrice a supplanté la logique utilitaire de la consommation débouchant ainsi sur une incessante recherche de nouvelles substances. Depuis la redécouverte des propriétés de l'aloe vera jusqu'aux L-Casei Defensis, notre intériorité doit être protégée, tout comme notre apparence extérieure doit rester belle et préservée. La plupart des consommateurs ignorent ce que sont exactement les radicaux libres ou l'oxygène pur, mais ils ont aveuglément confiance en leur utilité pour les soins personnels. Cette foi permet une invention constante de termes dont la puissance est d'autant plus réelle qu'elle est imaginée. Cela explique pourquoi le marché se trouve saturé de terminologie scientifique - parfois déconcertante - à l'instar des marques d' " ADN marine », ou de produits qui ne relèvent manifestement pas du cadre des cosmétiques, tels que les détergents, en insistant sur leur caractère «naturel » comme un puissant appel magico-publicitaire.

Une nature toujours plus dépendante des industries qui, avec ses profits considérables, nous fournissent tout ce que nous pouvons aimer ou désirer, depuis les boucles de cheveux "naturelles ", jusqu'à l'élimination de la cellulite avec des « résultats visibles », des crèmes raffermissantes pendant la grossesse ou puerpérales, ou encore des régimes spécifiques et des injections avec effets garantis de remise en forme physique. Cette offre progressive se base sur la facilité avec laquelle nous pouvons obtenir ces produits, leur faible risque et la rapidité de leurs effets. En minimisant le coût, l'effort et le temps réel qu'ils impliquent, la publicité parvient à consolider une fiction dans

11 Teresa DE LAURETIS, « La tecnología del género », Revista Mora, 2 (1996), p. 6-34. 
laquelle disparaissent les différences de classe, les contradictions entre la vie professionnelle et familiale, et jusqu'à la spécificité du corps féminin homogénéisant ainsi toutes les femmes. Ceci est la base d'une identification collective qui nous conduit à croire que nous pouvons «être » comme les modèles figurant sur les annonces ou dans les médias dont l'âge et la taille renvoient à l'écart à la réalité.

Ce qui nous est vendu pour sa facilité est plutôt un effort quotidien pour se conformer aux normalisations de la mode Le caractère anodin de ces usages, causant des dommages physiques à la population jusqu'à atteindre l'anorexie, et la rapidité se métamorphose en frustration lorsque nous ne parvenons pas à obtenir ce que nous recherchons. Les modèles se diffusent de sorte que, dans la confusion, nous pouvons choisir d'acheter la taille que nous désirons en croyant que nous sommes plus minces. Les magasins de vêtements utilisent des miroirs déformants qui affinent la silhouette corporelle afin de maintenir la chaîne de consommation. La spécialisation dans les grandes tailles met l'accent sur les nouvelles hiérarchies de corps. La confiance "magique» repose sur le fait de se tenir éloigné de tout embonpoint. Paraître âgé, ne pas se conformer au poids idéal ou ne pas se soucier de son apparence vestimentaire se trouvent être des indicateurs d'appartenance aux classes inférieures, aux groupes ethniques minoritaires, aux catégories marginalisées stigmatisées par leur rejet même de cette hégémonie. Et dans une société comme la société espagnole, où la crise économique a polarisé la structure sociale, la visibilité des «riches » par rapport aux autres utilise la beauté comme un élément de légitimation.

L'égalité présumée entre les femmes que promet le nouveau système de techno-beauté est devenue un facteur social plus important qu'il y a quelques décennies. «Être jeune» est une exigence esthétique que certaines, seulement, peuvent se permettre. L'irréversibilité de la chirurgie a été assouplie pour multiplier ses opérations jusqu'aux parties les plus intimes du corps - des gonflements, réductions ou surélévations des seins, jusqu’à la reconstruction des mamelons ou des voies génitales -, et les femmes sans ressources propres ou familiales suffisantes se sentent toujours plus éloignées des options dont bénéficient les catégories les plus riches.

Les formes de domination s'exercent à travers la différence esthétique liée à l'âge ; ainsi, notamment, les femmes les plus âgées sont conduites à 
paraître adolescentes par des opérations chirurgicales, des régimes permanents et de coûteux gymnases, alors que les plus jeunes, obsédées par les marques, embellissent leur corps soumis à la comparaison frénétique qu'engendre la concurrence - une concurrence qui altère les relations intraet intersexuelles avec un impact majeur dans le cas des femmes appartenant aux catégories opprimées. Et dans ce système, la fragmentation des corps permet de « croire et de créer » une illusion d'horizontalité sociale : « toutes peuvent être belles » ou au moins mettre en valeur les caractéristiques de leurs corps qui correspondant le mieux à l'imaginaire social (des seins parfaits, les yeux avec de longs cils, des jambes fuselées).

Pour beaucoup de femmes, le fait de privilégier les soins qu'elles s'apportent à elles-mêmes au détriment de leurs responsabilités sociales d'apporter un soin aux autres - surtout s'agissant des « charges de famille »est lié au désir de « jouissance » et d'entretien de leur propre corps. Il s’agit de «ne pas s'abîmer ». Le contraste est frappant avec les autres femmes, celles qui sont en dehors du système hégémonique, pour qui avoir des enfants reste l'un des moyens de réalisation, où le corps agit comme un support et une limite en fonction de leurs particularités. À la fin du $\mathrm{Xx}^{\mathrm{e}}$ siècle et durant la première décennie du $\mathrm{XXI}^{\mathrm{e}}$ siècle, le taux de natalité a diminué en raison de l'accès tardif des jeunes à un marché pouvant leur offrir un travail stable, la préférence pour l'allongement de la période de la vie sans charge de famille renvoyant la grossesse à un âge plus avancé, ainsi que les nouvelles formules pour avoir des enfants. Soumises au poids croissant de la médecine occidentale et des politiques publiques qui ont changé les mécanismes de planification familiale pour de nouveaux modèles sanitaires de procréation, sous la supervision de professionnels qualifiés, il n'est pas surprenant que l'on rouvre des débats virulents sur l'avortement ou les droits des femmes, comme cela est arrivé en Espagne. La relation étroite entre les tendances démographiques, le contrôle de la procréation et les idéologies étatistes a été un élément récurrent des revendications féministes puisque le corps des femmes est l'objet sur lequel convergent ces trois facteurs. 


\section{Des soins extérieurs au soin personnel}

Dès que l'on a revendiqué l'égalité des droits entre les femmes et les hommes au XIX ${ }^{\mathrm{e}}$ siècle, l'expérience de l'enfantement - qui s'est traduite par la conscience chez certaines féministes d'être procréatrices - fut l'un des arguments sur lesquels la revendication s'est appuyée. Hubertine Auclert, opposant le pouvoir mortifère des hommes au pouvoir des femmes de donner la vie, affirmait ainsi :

Vous admettez le vote pour les despotes, les prêtres, les soldats, oiseaux de proie affamés de carnage. Vous les distinguez même dans la foule pour leur donner, avec le mandat de vous conduire, quelques fois celui de vous assassiner. Et aux femmes créatrices, aux femmes amies de l'humanité, vous refusez de donner voix au chapitre de vos destinées $!^{12}$

La poursuite du don de soins à autrui comme moyen de lui témoigner de l'affection, la conformité aux normes modelant le corps des femmes en tant que consacrées à «d'autres » ont été, tout à la fois, des éléments de soumission et de résistance $e^{13}$.

Le mouvement féministe s'est ancré, à cette époque-là, dans des actions de femmes qui combattaient pour l'amélioration de la situation sociale des plus vulnérables. Le soutien aux esclaves des États-Unis a suscité la lutte pour la citoyenneté des femmes malgré les obstacles qui les réduisaient avant tout au statut de mère-épouse. À cette époque, la fierté de ce qui avait été défini comme spécifiquement féminin avait, du côté de certaines associations de femmes, une traduction politique : les soins et la protection étaient des armes pour le progrès de la société. Dans cette valorisation, on ne recourrait pas aux arguments biologiques étant donné que de nombreuses femmes pauvres gagnaient leur vie grâce à l'allaitement ou à l'éducation des enfants des classes aisées. La nourrice et les gouvernantes occupaient une position privilégiée parmi les domestiques au service des classes moyennes. Pour beaucoup de bourgeoises, procréer n'empêchait pas de rechercher d'autres femmes pour remplir les fonctions qu'elles n'avaient pas l'intention

12 Hubertine AuCLERT, Rapport au $3^{\mathrm{e}}$ Congrès national ouvrier (Marseille, 20-31 octobre 1879).

13 Francine SAILlant, «Cuidados, deseos vinculares y utopias terapeúticas: un análisis del concepto de caring ", in J.M. Comelles, M.A. Martorell \& M. Bernal (eds), Enfermería y Antropología, Barcelona, Icaria, 2009, p. 189-218. 
d'assumer à cause du prestige social qu'elles devaient conserver. D'où la revendication d'une différence dans les modes de comportements qui séparaient les hommes et les femmes, pourvu que ce modèle de conduite s'éloigne de la biologie ou de la nécessité d'être une mère pour devenir une femme complète ou accomplie. La revendication d'une « maternité comme fonction sociale » s'est généralisée dans les pays européens tels que la France, l'Allemagne, l'Italie ou la Norvège et s'est implantée comme un modèle mixte dans de nombreux pays colonisés.

Contrairement aux lois philanthropiques contre la pauvreté ou aux lois de charité publique, l’État se consolida autour de l'adoption de mesures de sécurité sociale concernant les mères et les femmes en général ${ }^{14}$. Les craintes au sujet d'une augmentation de la population ont été contredites par une réalité radicalement opposée : la chute du taux de natalité depuis la fin du XIX ${ }^{\mathrm{e}}$ siècle. Les politiques de santé publique ont tenté de réduire l'importante mortalité infantile en diffusant des conseils sur les soins pendant la grossesse, en améliorant les conditions d'hygiène tout au long du processus de procréation et en recommandant d'atteindre une meilleure qualité de la santé des femmes. Beaucoup de femmes ont commencé à exiger un salaire pour les mères, y compris pour toutes celles qui réalisaient ces fonctions. Cependant, le taux de natalité continua de baisser au cours de la Première Guerre mondiale, la période de l'entre-deux-guerres et durant la Seconde Guerre mondiale.

La crise économique et les guerres concentrèrent les politiques de planification, comme dans le cas espagnol étudié par Mary Nash ${ }^{15}$, en imposant une idéologie nataliste survalorisant les familles par des incitations et de la propagande. Globalement, les aides et les subventions accordées étaient attribuées pour favoriser les intérêts de l'État ou ceux des conjoints par le renforcement de la dépendance des femmes à l'égard de leur mari. L'exaltation du père, travailleur et viril, capable de surmonter les obstacles au profit de sa famille, démontre le caractère autoritaire de la relation entre les

14 Gisel Bock \& Pat Thane, Maternidad y políticas de género, Madrid, Cátedra, 1991.

15 Mary NASH, «Pronatalismo y maternidad en la España franquista », in G. Bock \& P. Thane, op. cit, p. 279-307. 
États et les ménages dans un contexte historique et culturel donné. L’image de la mère femme, gardienne du foyer et femme heureuse fut également réinstituée en Espagne sous le régime franquiste comme l'analyse Osborne ${ }^{16}$.

La diminution du nombre de filles obéissait aux exigences d'un marché concurrentiel et à la transformation de certains États qui préféraient la qualité à la quantité des nouvelles générations. Contrairement aux économies où les fils/filles représentent une main-d'œuvre vitale, et les femmes une source d'alliances par les mariages, les exigences des années 1950 et 1960 ont déterminé des changements dans les priorités démographiques. Betty Friedan, en tant que thérapeute, a révélé des problèmes de santé inédits découlant d'une exaltation du rôle des femmes en tant que mères et épouses, sans possibilité de développer leurs propres besoins ou leur potentiel intellectuel. Son ouvrage, La Femme mystifiée (The Feminine Mystique) ${ }^{17}$, fut une référence des années 1960 pour alimenter les revendications centrées sur le contrôle de la sexualité, les droits à l'avortement, la liberté des modèles culturels et l'égalité dans l'accès à l'emploi. D'où la cristallisation d'une idéologie de la maternité comme un aspect « traditionnel », " dangereux », qui pourrait introduire un solide revers pour les droits acquis par les femmes ou que les revendications féministes s'acheminent vers des obligations cristallisant la parentalité et le partage des tâches ménagères.

Au sein de l'État espagnol, à partir des années 1970, le modèle de pureté des mœurs et de chasteté qu'avait imposé le régime franquiste a commencé à se fissurer. L'acquisition de droits liés au féminisme, tels que le divorce, l'avortement et l'accès aux nouvelles techniques de planning familial, a favorisé une vision renouvelée de la maternité contrairement à ce qui s'est passé dans d'autres pays, à mesure que la croissance économique et le changement des habitus familiaux se consolidèrent à partir des années 1990.

\section{La fragmentation magique de la citoyenneté}

En considérant les femmes comme des agents liés à la reproduction et aux soins, au travail domestique et à la sphère privée, on légitime leur silence ou

\footnotetext{
16 Raquel OsBorne (ed.), Mujeres bajo sospecha. Memoria y sexualidad: 1930-1980, Madrid, Editorial Fundamentos, 2012.

17 Betty Friedan, La mística de la feminidad, Gijón, Júcar, 1974.
} 
leur invisibilité. Ainsi se consolide sur un plan idéologique une vision biaisée par laquelle les hommes sont les sujets actifs de l'histoire et les seuls protagonistes du changement social. Les relations d'inégalité et d'exploitation entre les différents pays ont de profondes racines historiques ${ }^{18}$ qui sous-tendent et reproduisent les discours de supériorité de l'Occident. Les femmes sont utilisées, dans ces discours et ces pratiques, comme des signes de décadence ou d'immoralité des autres peuples. C'est à elles que l'on recourt pour illustrer l'oppression, la sauvagerie et le déclin économique; elles sont rendues responsables, dans les pays où elles immigrent, de maintenir les traditions culturelles, même illégales, de reproduire, auprès de leurs enfants ce qu'elles ont appris de leurs ancêtres et ce qui les relie à leur groupe d'origine.

Les différences qui dépendent du pays d'origine, de la position sociale, de l'année et des conditions d'insertion empêchent d'aborder une question comme la maternité dans différents collectifs de femmes de manière systématique. Il nous faut prendre en compte le fait, comme l'a noté Lila Abu Lughod ${ }^{19}$, que, historiquement, des ruptures et des changements complexes ont eu lieu dans les nations colonisées utilisant la maternité et la parentalité comme arguments pour légitimer l'ordre établi ou pour créer des espaces de résistance.

L'accès à des emplois fortement genrés, comme le travail domestique, montre que la conciliation, telle qu'elle est définie par la loi, prend un sens différent. Au sein de la diversité des activités, il est important de souligner celles qui sont regroupées sous le terme « soins », qui incluent des modèles spécialisés d'entraînement, d'ornement corporel, d'alimentation et d'hygiène des enfants, et/ou d'accompagnement et de soutien aux personnes âgées, lesquelles exigent fréquemment des mesures et des traitements entrant dans le cadre du travail social : soin des personnes souffrant d'un certain degré d'invalidité, régimes alimentaires ou médications typiques. Sans aucun doute, nous parlons d'un vaste univers d'actions protocolarisées, qui requièrent un ensemble de compétences spécifiques, clairement professionnelles lorsqu'elles se produisent dans la

18 Sophie BEssis, Occidente y los otros. Historia de una supremacía, Madrid, Alianza, 2002.

19 Lila ABu Lughod, Feminismo y modernidad en Oriente próximo, Madrid, Cátedra, 2002. 
sphère publique. Cependant, dans la sphère privée, ce n'est pas la qualification, mais l'affection ${ }^{20}$, qui permet de les accomplir. Dans ce domaine, le facteur déterminant est le lien entre celles qui procurent les soins et ceux qui en bénéficient - un lien contractuel de nature informelle vers lequel convergent les aspects qualitatifs différenciateurs de genres. Le maintien de la « féminité » avec toute sa charge naturalisante est le facteur qui confère non seulement la responsabilité des soins, mais encore l'obligation de leur bonne exécution.

L'affection et la connaissance s'associent par une relation d'engagement plaçant les femmes dans une position d'infériorité par rapport aux hommes - tant celles qui acquièrent par le mariage une position sociale enviable et du prestige en tant qu'épouses, que celles qui travaillent pour d'autres familles. Ce sont elles qui doivent alors ordonner un espace familial qu'elles considèrent comme le leur ${ }^{21}$. Les familles monoparentales ou les femmes seules n'échappent pas à ce principe fondamental de structuration sociale. Les femmes assument et reproduisent un modèle générique qui, en dépit des changements dans le travail et la sphère domestique, maintient le « don de familiarité » dans le corps féminin.

La séparation des espaces et l'attribution de rôles opposés selon le système sexe/genre détermina l'accès différentiel à la citoyenneté des hommes et des femmes. Comme la sphère publique correspond à l'espace prestigieux et aux hommes, la charge symbolique de ces activités se trouve fortement valorisée. De l'homme, l'on exige des capacités de prise de décisions, de gestion ou de commandement, étant donné que sa fonction principale est de fournir au groupe domestique les ressources suffisantes pour sa subsistance.

Alors que se déploient des politiques d'assistance spécifiques pour les femmes migrantes, les États ont mis au point des politiques natalistes bénéficiant à ces femmes avec la pleine reconnaissance de leur citoyenneté et la position de classe qui garantissait leur droit d'être mère. Dans le cas de l’État espagnol, ce fut le gouvernement Zapatero qui attribua une somme aux naissances en période de prospérité économique apparente. Ce chèque bébé supposait un versement non imposable de 2500 euros entre 2007 et 2010, auxquels s'ajoutaient 1000

20 Susana NAROTZSKY, Mujer, mujeres, género, Madrid, Consejo Superior de Investigaciones científicas, 1995.

21 Àngeles DE LA CONCHA \& Raquel OsBoRne (eds), Las mujeres y los niños primero: discursos de la maternidad, Barcelona, Icaria, 2004. 
euros s'il s'agissait de femmes célibataires, de familles nombreuses ou d'enfants handicapés. De 2007 à 2010, la magie de cet argent supplémentaire signifia une « aide » à la procréation sans distinctions de classe.

\section{En forme de réflexion : économie, néo-libéralisme, sacralisation de la maternité et droits fragmentés}

La prolifération des banques de sperme, des techniques de fertilisation et d'adoptions internationales placent la maternité et les soins dans un contexte radicalement différent du passé. La technologie et l'argent pour accéder à ces « biens » ne sont pas étrangers à ces nouveaux processus de demande de main-d'œuvre féminisée par lesquels les travaux domestiques, les soins aux enfants ou aux anciens fonctionnent comme des niches d'emplois traditionnellement dévolus aux femmes appartenant aux classes laborieuses. Les nouveaux modèles affectent également l'établissement de relations entre les hommes et les femmes. Les unions de fait, la reconnaissance juridique du mariage homosexuel et le nombre croissant de familles monoparentales ou de femmes célibataires qui assument la maternité avec l'aide d'amis et de parents, ou l'augmentation des divorces reformulent le sens des identités familiales, de la maternité et de l'élevage des enfants.

Dans ces nouvelles conditions, la maternité retrouve un poids idéologique prenant de l'importance comme expérience personnelle. Loin de ses connotations biologisantes, notre société a confiance en la technologie et envers les choix économiques, légaux ou non, qui offrent certaines conditions pour la procréation et le soin aux enfants. Le problème est que les différences de classe et de position sociale entre les femmes s'aiguisent. À l'intérieur ou à l'extérieur, les possibilités d'embauche de "mères porteuses », de donner la charge des enfants aux femmes les plus pauvres, de surveiller celles qui sont déjà sous contrat pour du soin ou du travail domestique, de disposer des capitaux nécessaires pour assurer les techniques de reproduction, mettent en relief l'émergence de nouvelles situations où l'inégalité n'est pas résolue ${ }^{22}$.

22 Emma MARTín \& Assumpta SABUCO, Las mujeres en la globalización: el nuevo tráfico de alianzas y mercancías, Caracas, Venezuela, 2006. 
La demande de services de santé reproductive est très rentable pour de nombreuses entreprises, tout comme pour les institutions, les associations et les intermédiaires dans les processus d'adoption qui marchandisent « le désir d'être parents » sans une réglementation stricte par les États. Tout au contraire, les accords interpersonnels, les pratiques qui deviennent de la législation ambiguë en ce qui concerne les droits de reproduction sont criminalisés.

Les nouvelles représentations de la maternité s'ajustent aux conditions du néo-libéralisme patriarcal : les coûts d'adoption deviennent moins chers dans le cas des filles, les ovules ne méritent pas la même valeur marchande que les banques de sperme ; les effets et techniques sur le corps des femmes en processus de fécondation sont définis depuis le sacrifice, non depuis le risque. Il n’est dès lors pas étonnant que, au sein des différentes disciplines scientifiques, augmente l'intérêt pour les processus de reproduction biologique à partir des années 1970 et 1980, en particulier pour les soins obstétricaux et les conséquences de la médicalisation ${ }^{23}$.

Les analyses sur les savoirs féminins relatifs au corps des femmes, sur les effets de la dépréciation de ces derniers et l'accent mis sur le contrôle médical de la reproduction, l'imposition de techniques rapides dans le contexte sanitaire - avec l'augmentation des césariennes dans les naissances programmées - et les instructions normatives dans les processus de la mise

23 Paola TABET, «Fertilité naturelle, reproduction forcé », in N.-Cl. Mathieu (éd.), L'arraisonnnement des femmes. Essais en anthropologie des sexes, Paris, École des hautes études en sciences sociales, 1985.

Ann OACKLEY, The captured womb: a history of the medical care of pregnant women, Oxford, Basil Berstein, 1986.

Verena STOLCKE, «Las nuevas tecnologías reproductivas. La vieja paternidad », in C. Amorós et al., Mujeres: ciencia y práctica, Madrid, UCM, 1987, p. 87-129.

Rayna RAPP, Testing Women, Testing the Fetus: the Social Impact of Amniocentesis in America, New York - London, Routledge, 2000.

Marilyn STRATHERN, Reproducing the future. Anthropology, kinship and the new reproductive technologies, Manchester, Manchester University Press, 1992.

Susana NAROTZSKY, op. cit.

Mari Luz Esteban, "La maternidad como cultura ", in E. Perdiguero Gil \& J.M. COMELLES (eds), Medicina y cultura: estudios entre la antropología y la medicina, Barcelona, Bellaterra, 2000. 
au monde et de l'élevage consolident une vision fragmentaire donnant le primat au fœetus et au bébé sur le corps de la mère ${ }^{24}$.

Pour certains auteurs, il en résulterait un nouveau regard sur le corps des femmes enceintes et leurs relations avec le fœtus. Un premier modèle serait marqué par la prédominance d'une représentation de la fusion mère-enfant où les besoins en soins et attentions découlent des dangers qui peuvent surgir pour la survie du fœtus. L'ignorance du processus implique la poursuite inquiète et sans relâche des connaissances faisant autorité pour assurer le succès de la procréation avec des lectures, l'assistance à des conférences, des séances d'information ou des conseils médicaux. Le deuxième modèle relève d'une représentation en termes d'invasion ou de parasitisme par laquelle le rapport de la femme à son propre corps serait marqué par l'étrangeté et le danger pour sa santé, mettant alors l'accent sur le respect des soins pour prévenir les états de carence ou des dommages physiques irréversibles. La peur et l'insécurité face à un corps scindé encouragent également la recherche de solutions professionnelles, y compris pour traiter les aspects émotionnels et les problèmes psychologiques du processus. Dans les deux cas, nous assistons à une nouvelle fragmentation du corps féminin.

Pour beaucoup de femmes des sociétés développées et des classes moyennes, la forme de résistance à ces modèles implique une défense obstinée du caractère naturel de la reproduction, une revendication constante du pouvoir que suppose engendrer-soigner et une revitalisation des pratiques traditionnelles telles l'accouchement à domicile ou sans aides palliatives à la douleur. En comprenant que la médicalisation croissante de la grossesse suppose sa définition comme maladie, les pratiques alternatives au niveau individuel ont augmenté, non sans le soutien d'associations d'aide mutuelle à l'accouchement, avec l'allaitement prolongé et même à travers des déclarations institutionnelles telles que celle de Fortaleza, en 1985, où l'OMS a défendu cette conception naturelle en déclarant les femmes enceintes en bonne santé.

Dans cette affirmation du rôle des femmes comme sujets actifs face aux institutions médicales et d'État, la nouvelle idéologie sanctifie, d'une part, la

24 Robbie DAvis-FloyD \& Elizabeth Davis, «Intuition as authoritative knowledge in midwifery and homebirth ", Medical Anthropology Quarterly, 10, 2 (1996), p. 237-269. 
maternité comme l'une des expériences qui définissent le plus le féminin et revendique le rôle des hommes en tant que partenaires de «l'expérience la plus cruciale de l'humanité ». Le rôle de la paternité est donc redéfini en renforçant l'hétéronormativité et l'hégémonie du couple dans le domaine de l'affectif. Le droit au mariage entre personnes du même sexe est une nouvelle désignation de la valeur «modernisatrice, progressiste » d'un Occident abstrait dans lequel les pays qui régulent cette pratique sont présentés légitimement en tant que parties prenantes.

Mais les changements de ces nouvelles parentalités peuvent résulter de l'aspiration d'hommes célibataires à travers l'exaltation de la «nécessité » de la progéniture. Les hommes - fiancés ou maris - sont toujours plus nombreux à assister régulièrement aux consultations prénatales, aux cours de respiration et de motricité, à l'apprentissage des formes adaptées de soin aux enfants dès la naissance, et assistent à l'accouchement en tant que participants. Ou que l'on explique le droit à louer légalement un ventre comme une aspiration au désir «naturel » d'être père. Un désir accru par l'impossibilité, en cas d'affinité sexuelle entre hommes. Pour les couples de lesbiennes, c'est tout simplement le désir maternel qui est mis en sourdine, ignoré dans la genèse reproductive « des femmes ».

L'exaltation de cette nouvelle "procréation naturelle » justifie que des femmes entre 40 ans et 50 ans préfèrent donner naissance à la maison, sans médiations techniques ou pharmacologiques, comme un rejet explicite de la technologie. Ou que l'on engendre des enfants qui seront ensuite éloignés de la patria potestad comme dans le cas d'une femme de Huelva ayant donné naissance à des jumeaux à 67 ans, alors qu'elle avait été légalement séparée d'une fille conçue à 50 ans. Le caractère exceptionnel du moment justifie les enregistrements de l'événement en format numérique ou l'accumulation de "preuves » sur les changements corporels vécus par les mères et leurs enfants rendus possibles par une technologie qui progresse rapidement dans l'amélioration des images en trois dimensions et dans la possibilité d'enregistrer des sons ou des mouvements du fœtus.

Cependant, les actuelles conditions économiques et salariales rendent plus difficiles les soins et l'éducation des enfants en dépit des prestations et indemnités temporaires octroyées par les États tant pour la maternité que pour la paternité. L’incompatibilité des horaires du travail avec ceux de l'école 
génère une recherche de temps ou de réseaux, et d'institutions spécifiques, ou encore des emplois permettant de les concilier. L'allongement de l'espérance de vie, le fait de disposer de temps, l'affection et l'épargne ont converti les grands-parents - père et mère des géniteurs - en personnes chargées de ces fonctions de soin et d'éducation. Mais la plupart du temps, ce sont des femmes d'autres classes sociales, de groupes ethniques défavorisés ou provenant de pays pauvres, qui sont engagées pour remplir ces fonctions. Pour les unes, les idéaux de beauté et de maternité deviennent accessibles alors que d'autres, sans capital économique suffisant, s'en éloignent. Dans les deux cas, nous assistons à une réinvention magique de la nature - malgré l'artificialité technologique qui la constitue - envahissant nos représentations du corps et favorisant les intérêts dominants tant sur le plan politique qu'économique.

Les investissements et les avantages monétaires, l'augmentation du nombre des professionnels de la beauté et de la santé - notamment en matière de procréation -, la multiplication des techniques et des experts en pratiques alternatives pour nos soins, tout comme la conjonction avec les intérêts de l'État nataliste, comportent des risques graves : le renforcement des modèles de genre inégalitaires, la réinvention et l'appropriation de la nature culturelle de notre corps, ainsi que le développement de la séparation entre les secteurs sociaux. Ces processus accentuent également une confrontation croissante entre les droits fondamentaux. D'une part, la modernité de la reconnaissance des mariages entre homosexuels se diffuse et se popularise dans les pays "avancés ", alors que, simultanément, les débats autour de l'avortement s'ouvrent à nouveau. Comme le précise Blanca Rodríguez ${ }^{25}$, ces lois sont insérées dans un nouveau renforcement de l'inégalité entre les femmes et les hommes. Dans le cas de l'Espagne, la proposition du ministre Gallardón en 2011 était fondée sur la défense de la vie du fœtus contre le droit des femmes à décider. Supposer que ce sont deux entités différentes et antagonistes, qui ne font pas partie du même processus de gestation, fragmente la réalité corporelle afin de lancer des querelles sur le moment où « cette vie » commence et sur qui doit décider.

25 Blanca RodríGuez, « Gender in Constitutional Discourses on Abortion: Looking at Spain from a Comparative Perspective », Social \& Legal Studies, 25, 6 (2016), p. 699-715. 
Ce démembrement des corps change sensiblement la façon dont on pense et régule les droits de l'Homme. Pour la droite conservatrice, la hiérarchisation des valeurs, liées à la religion dans le cas espagnol, explique qu'ait été proposée une loi pour la défense de la vie s'opposant au droit des femmes de décider. Avec la maternité de substitution c'est, en revanche, le droit au libre choix sans que le sexe n'intervienne qui est postulé face à une femme qui n'est plus mère. Comme le signale Verena Stolcke ${ }^{26}$ nous devons interroger et montrer les relations complexes établies entre le genre et la parenté.

L'insertion de la maternité et de la paternité dans le domaine du beau et dans les mesures prises par des politiques qui, d'une façon plus ou moins spectaculaire, rentabilisent leurs idéologies avec des chèques-bébés, la promesse de protéger les non-nés ou avec des argumentations en faveur de la maternité de subrogation établit un «beau désir de vie » qui repose sur cette dislocation ethnocentrique des corps : d'un côté, entre mère et fœus considérés dans les législations sur l'avortement comme unités corporelles distinctes, unités dont les États deviennent les garants des soins ; et, d'un autre côté, dans la «gestation pour autrui » avec le primat du contrat sur les désirs ou les sentiments de la mère porteuse transformée en réceptacle sans droits, mais avec une charge temporaire de soins à donner. Dans les deux cas, le corps féminin en gestation est morcelé en différentes parties à statut juridique variable.

Par cette convergence de l'esthétique et du procréatif, il n'est pas surprenant que les femmes organisent un grand nombre de leurs propositions dans un cadre protestataire où le corps fragmenté provoque des réactions de colère, comme dans le cas de la manifestation du Coño Insumiso (le Con insoumis) avec ses revendications juridiques, événement par lequel une image de vulve fut arborée durant la fête du travail, simulant ainsi les processions andalouses. Les requêtes réitérées de l'Association des avocats catholiques signalaient que cette utilisation relevait de l'insulte aux sentiments religieux. Ou l'utilisation du sang comme symbole d'une appropriation féminine de la

26 Verena STOLCKe, «Homo clonicus:¿entre la naturaleza y la cultura? », CAMPOSRevista de Antropologia Social, 11, 2 (2010).

Verena Stolcke, "Que tem a ver o gênero com o parentesco? ", Cadernos de Pesquisa, 44, 151 (2014), p. 176-189. 
double et simultanée valeur des menstrues à laquelle s'opposent des significations négatives associées au dégoût et à la honte ${ }^{27}$.

Telles sont quelques-unes des nouvelles conditions de la soumission basées sur la fragmentation corporelle auxquelles nous cédons magiquement en comptant sur la technologie intelligente, pour « notre propre bien-être ». En contrepoint, le féminisme « active » des réponses contestataires face aux menaces les plus irrépressibles de la mondialisation.

\section{Traduit de l'espagnol par Salvador Juan (traduction relue par Assumpta Sabuco i Cantó)}

\footnotetext{
* Ce texte est la traduction de la version actualisée de l'article d'Assumpta SABUCO I CANTÓ, «Los cuerpos fragmentados de la globalización: de la estética a la maternidad », in C. Gregorio Gil \& M.P. Castañeda Salgado (eds), Mujeres y hombres en el mundo global. Antropología Feminista en América Latina y España, Siglo XXI Editores, 2012, (c) D.R. Universidad Nacional Autónoma de México.
}

27 Assumpta SABuco, «Artivismo sangriento: de Pilar Albarracín a los nuevos movimientos feministas ", in III Encuentro Mil Formas de Mirar y Hacer: Artes y Movimientos sociales (Sevilla, 28-29 de septiembre de 2016), Proyecto Atalaya, Dirección General de Universidades de la Consejería de Economía y Conocimiento de la Junta de Andalucía, 2017. 
Hors-dossier 
